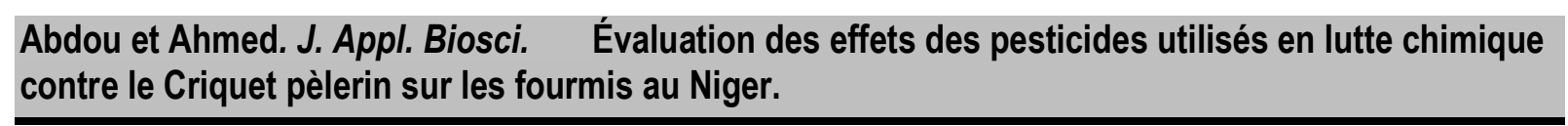

Journal of Applied Biosciences 88:8144- 8153

ISSN 1997-5902

\title{
Évaluation des effets des pesticides utilisés en lutte chimique contre le Criquet pèlerin sur les fourmis au Niger.
}

\author{
Mamadou Abdou' ${ }^{1}$ et 2 Mazih Ahmed \\ ${ }^{1}$ Centre National de Lutte Antiacridienne, BP 2219, Niamey, Niger, GSM 00227 96508175, \\ 2 Institut Agronomique et Vétérinaire Hassan II, Département de Phytiatrie, B.P. 18/S - 80 000, Agadir (Maroc) \\ Courriel: abdoumamadou@yahoo.fr
}

Original submitted in on 23rd January 2015. Published online at www.m.elewa.org on $30^{\text {th }}$ April 2015 http://dx.doi.org/10.4314/jab.v88i1.3

\section{RÉSUMÉ}

Objectif : Les traitements chimiques constituent encore la principale méthode utilisée pour lutter contre le Criquet pèlerin en période de recrudescence ou d'invasion. Les pesticides ne sont pas très spécifiques dans la plupart des cas, ils peuvent avoir des effets négatifs sur les composantes biotiques et même abiotiques. En effet, une des conséquences environnementales majeures de la lutte contre les locustes, c'est l'utilisation croissante et récurrente des pesticides conventionnels, notamment dans des écosystèmes désertiques tels que la vallée du Tafidet au Niger. L'objectif de notre étude conduite dans des conditions naturelles est d'évaluer l'effet écologique et toxicologique du chlorpyriphos-éthyl et du fénitrothion, pesticides organophosphorés les plus utilisés dans la lutte contre le Criquet pèlerin au Niger.

Méthodologie et résultats : L'analyse de la variance est choisie pour effectuer une comparaison statistique basée sur le principe BACl (Avant-Après-Contrôle-Impact). Le dispositif expérimental utilisé est le plan en blocs aléatoires complets avec trois répétitions. Les unités expérimentales sont des carrés de 16 hectares, les traitements sont constitués de chlorpyriphos-éthyl à la dose de $225 \mathrm{~g} \mathrm{~m}$.a/ha ; de fénitrothion à la dose de 450 $\mathrm{g}$ m.a/ha. Les parcelles témoins n'ont reçu aucune pulvérisation de produit. Les traitements chimiques sont effectués en couverture totale à l'aide d'un appareil porté à la main muni d'un disque rotatif (Micro-Ulva ${ }^{\circledR}$ ). Les expérimentations ont été conduites dans la vallée du Tafidet située sur le versant oriental des massifs de l'Aïr au Niger. Les résultats obtenus ont mis en évidence l'impact négatif du chlorpyriphos-éthyl et du fénitrothion (organophosphorés) sur les fourmis. En effet, les fourmis ont été particulièrement sensibles aux doses des pesticides utilisés. De ce fait, les fourmis pourraient servir d'indicateurs biologiques de la présence d'une substance xénobiotique dans un environnement, puisque le taux de réduction de leur population dépasse $98 \%$ en présence des pesticides, 12 jours après les traitements phytosanitaires. De manière générale, les réductions numériques des populations des insectes ont été plus fortes dans les deux premiers intervalles après les applications (1-12 jours et 16-24 jours). Par contre, dans le troisième intervalle de temps, c'est-à-dire, 28-40 jours après les traitements, les effets des différents pesticides sont très faible. Le nombre de fourmis capturées dans les différentes unités expérimentales a diminué y compris dans les parcelles non traitées.

Conclusion et implication des résultats : De manière générale, les résultats de cette étude ont mis en évidence que les pesticides utilisés en lutte contre le Criquet pèlerin ne sont pas spécifiques aux acridiens. Ils ont des effets néfastes sur les insectes dits écologiquement utiles comme les fourmis. Les fourmis pourraient servir d'indicateurs biologiques de la présence d'une substance xénobiotique, notamment les insecticides chimiques, 


\section{Abdou et Ahmed. J. Appl. Biosci. Évaluation des effets des pesticides utilisés en lutte chimique contre le Criquet pèlerin sur les fourmis au Niger.}

dans un environnement, puisque le taux de réduction de leur population dépasse les $98 \%$ à 12 jours après les traitements.

Mots clés : Lutte Criquet pèlerin, pesticides organophosphorés, évaluation, effet, fourmis, Niger.

\section{ABSTRACT}

Objective : Chemical treatments still constitute the main method used to control desert locust during the invasion periods. These pesticides are not specific and they can have negative effects on the biotic and abiotic environment components. The major environmental consequences of the desert locust control by using of the conventional pesticides can be drastic, especially in ecologically fragile desert ecosystems such as the Tafidet valley of Niger. The goal of this study conducted under natural conditions, was the assessment of the ecological effect of Chlorpyrifos ethyl and Fenitrothion, the most widely used organophosphate pesticides in desert locust control in Niger.

Methodology and results: To assess the impact of pesticides on the relative abundance on wild bees before and after spraying, yellow trap methods were used. The analysis of the variance was used to carry out a statistical comparison based on BACl (Before-After-Control-Impact) method. The experiments were carried out in a randomized complete block design with three replications. The experimental units had an area of 16 ha each, the treatments consisted of Chlorpyriphos ethyl at the rate of $225 \mathrm{~g}$ a.i. /ha ; Fenitrothion at the rate of $450 \mathrm{~g}$ a.i. / ha. Unsprayed plots were used as controls. Chemical treatments were carried out in total cover using a battery driven hand-held spinning disk sprayer (Micro-Ulva ${ }^{\circledR}$ ). The field trial was carried out in the valley of Tafidet located in the eastern side of Air Mountains in Niger. The results demonstrate the noxious effect of Chlorpyriphos ethyl and Fenitrothion (organophosphate pesticides) on the wild bees. It was shown that wild bees were particularly sensitive and can be used as biological indicators of the presence of a xenobiotic substance in an environment, since the reduction ratio of their population exceeded $98 \%$ in the presence of these pesticides, 12 days after treatment application. In general, the numerical reductions of the populations of these insects were great in the first two intervals after the applications (1-12 days and 16-24 days). In the third interval of time (28-40 days after the applications), the effect of pesticides was low. In this time interval, the total catches of ants were very low in all the treated plots and untreated plots.

Conclusion and the implication of results: In general, the results of this study showed that pesticides used to control desert locust are not specific to locusts. They have noxious effects on ecological useful insects like ants. Ants could serve as biological indicators of the presence of a xenobiotic substance, in particular chemical insecticides, in an environment, because the reduction of their population exceeds the $98 \% 12$ days after treatments.

Keywords : control desert locust, organophosphorus pesticides, assessment, effect, ants, Niger

\section{INTRODUCTION}

Les productions agricoles destinées à la consommation humaine ou animale ont besoin d'être protégées contre des ravageurs, qui sont susceptibles de provoquer des dégâts souvent irréversibles. Dans certaines parties du monde comme l'Afrique, l'Amérique du Sud ou l'Asie, les pertes peuvent représenter jusqu'à $48 \%$ des récoltes (Thiollet-Scholtus, 2004). Dans les zones arides et semi-arides d'Afrique et d'Asie, les sauteriaux et les locustes notamment le Criquet pèlerin (Schistocerca gregaria Forskål, 1775)
(Orthoptera : Acrididae) en période d'invasion ou de recrudescence, prennent une part active dans la déprédation des cultures et des zones de pâturage (Krall, 1994 ; Kogo et Krall, 1997 ; Bashir et al., 1998; FAO/PAM, 2004). La problématique du Criquet pèlerin, notamment en période d'invasion (présence d'essaims et des bandes larvaires) est traitée comme une priorité nationale dans les pays affectés (Lecoq, 2001). Par exemple, la production agricole de 2004 a été sévèrement affectée par l'invasion du Criquet pèlerin et la sécheresse dans 


\section{Abdou et Ahmed. J. Appl. Biosci. Évaluation des effets des pesticides utilisés en lutte chimique contre le Criquet pèlerin sur les fourmis au Niger.}

les régions Nord du Niger engendrant des poches d'insécurité alimentaire (FAO/PAM, 2004). Pour lutter contre les augmentations d'effectifs acridiens, le moyen de lutte le plus utilisé est le recours à des pesticides de synthèse. Depuis l'interdiction des pesticides organochlorés au début des années 1970, du fait de leur persistance trop importante dans les milieux naturels et des risques de bioaccumulation dans les chaînes trophiques (Römbke et Moltmann, 1996), de nouvelles molécules ont fait leur apparition. Le Niger utilise annuellement environ 130000 litres (moyenne pour la période 1994-03) de pesticides de synthèse (organophosphorés) pour lutter contre les sautériaux et le Criquet pèlerin en période de fortes résurgences ou de recrudescences (FAO, 1998, 1997). En plus de leur coût relativement élevé, les pesticides de synthèse ne sont pas sans risque pour la santé humaine. Ainsi, les personnes sont également susceptibles d'être intoxiquées par ces pesticides de synthèse. En effet, selon l'Organisation mondiale de la santé (OMS/PNUE, 1989), chaque année un million de personnes est intoxiqué accidentellement par des pesticides dans le monde et environ 20000 en périssent. Si l'on ajoute les cas intentionnels (il s'agit surtout de suicides) on arrive à 3 millions d'empoisonnements, dont 220000 morts (Levine, 1991). L'exposition du fœtus in utero à certaines périodes de la grossesse peut conduire à un avortement spontané, des retards de croissance et des malformations. Ainsi, une étude réalisée par Santé Canada a montré que le risque de fausse couche et de prématurité était plus grand dans les familles dans lesquelles le père avait manipulé certains pesticides. Le risque d'accouchement prématuré était de 1,7 à 2,4 fois plus élevé si le père avait manipulé des pesticides comme l'atrazine, le glyphosate ou des organophosphorés (Arbuckle et al., 2001). L'enquête de Clavel (1996), réalisée chez des fermiers propriétaires et des ouvriers agricoles s'adonnant à la culture de diverses céréales, de fourrage, de fruits et légumes, à l'élevage de gros et petit bétail, aboutit à soutenir l'argument selon lequel les OPs jouent un rôle dans le développement de la leucémie à tricholeucocytes. . Les études sur les effets désastreux ou délétères des pesticides de synthèse sur la faune non-cible étant très nombreuses, seules quelques-unes sont citées sans souci d'exhaustivité. Plusieurs auteurs rapportent que les insecticides tels que le carbaryl et le malathion (Roger et al., 1991), le malathion et le diazinon (Dinkins et al., 1971), le diazinon (Whalon et Elsmer, 1982) et la deltaméthrine (Thomas et al., 1990), ont des effets négatifs significatifs sur les populations des prédateurs en général. Aux États-Unis, l'intoxication par les pesticides serait responsable de plus de $20 \%$ des pertes de ruches d'abeilles domestiques et de pollinisateurs sauvages (Pimentel et al., 1991). Une étude menée par David (2001) sur les abeilles domestiques (Apis mellifera) montre qu'une concentration de $10 \mu \mathrm{g}$ d'imidaclopride (famille des chloronicotinyles) a un effet répulsif, alors que ces insectes jouent un rôle primordial dans la pollinisation des espèces végétales et dans la production en miel qui est une source énergétique importante (Roubik, 2002; Zimmermann et al., 2006 ; Liu et al., 2007). Depuis la Conférence des Nations Unies sur l'Environnement Humain (CNUEH), tenue en 1972 à Stockholm (Suède), et surtout la Conférence des Nations Unies sur l'Environnement et le Développement (CNUED), tenue en 1992 à Rio de Janeiro (Brésil), les questions environnementales sont ainsi devenues une composante majeure dans la gestion des invasions du Criquet pèlerin. C'est dans ce contexte que la Commission chargée de lutte contre le Criquet pèlerin à travers la FAO a financé cette étude de 2004-06. L'objectif de la présente étude est donc, d'évaluer en milieu réel, l'effet du chlorpyriphos-éthyl et du fénitrothion (pesticides organophosphorés) sur les fourmis qui jouent un rôle écologique important dans la vallée du Tafidet (versant oriental des massifs de l'Aïr). En raison de leur importance numérique, les fourmis jouent de grands rôles dans les forêts qu'elles occupent (Savolainen et al., 1989). Les fourmis sont de très importants prédateurs d'insectes et autres invertébrés (Niemelä et al., 1992, Punttila et al., 2004). Elles peuvent contribuer à limiter les populations d'insectes ravageurs comme les chenilles de papillons ou d'hyménoptères par exemple lors de pullulations (Karhu, 1998). Jusqu'à présent, plusieurs études évaluent les risques phytosanitaires pour la faune non-cible par la 


\section{Abdou et Ahmed. J. Appl. Biosci. Évaluation des effets des pesticides utilisés en lutte chimique contre le Criquet pèlerin sur les fourmis au Niger.}

mortalité mesurée après exposition d'insectes en laboratoire, or il existe un décalage important par

\section{MATÉRIEL ET MÉTHODES}

Site de l'étude: La vallée de Tafidet $\left(18^{\circ} 09^{\prime} 16 \mathrm{~N} / 09^{\circ} 30^{\prime} 52 \mathrm{E}\right)$ est située à environ $300 \mathrm{~km}$ au nord-est d'Agadez. Cet oued est alimenté par les massifs montagneux du versant Est de l'Air, le Takaloukouzet dont le point culminant est à $1295 \mathrm{~m}$. II constitue un des habitats permanents de reproduction estivale et de grégarisation du Criquet pèlerin. Le versant oriental de l'Air a fait l'objet de quelques études tant sur le plan floristique (Giazzi, 1996, 1993 ; Anthelme et al., 2007, 2006) que faunique, notamment la faune sauvage (Giazzi, 1996). En revanche, les données sur les insectes d'utilité écologique tels que les pollinisateurs, les coléoptères prédateurs et les hyménoptères parasitoïdes, issues de cet écosystème désertique sont à notre connaissance rares. C'est d'ailleurs l'un des points qui a sous-tendu le choix de cette zone pour conduire cette étude d'une part et, d'autre part, cet écosystème désertique fait partie de la Réserve Naturelle Nationale de l'Aïr et du Ténéré (RNNAT), classée patrimoine mondial de l'UNESCO en 1991 (UNESCO, 1996). Le zonage agro-climatique du Niger, classe la région d'Agadez, donc notre site d'étude, dans la zone saharienne, caractérisée par une pluviométrie annuelle normale inférieure à $150 \mathrm{~mm}$ et une saison de pluies normale qui dure à peine un mois, si bien que l'agriculture non irriguée est presque impossible (DMN, 2005). Les hauteurs moyennes de pluies enregistrées (2004-2006) dans l'oued du Tafidet, indiquent une répartition temporelle très irrégulière. Le mois le plus pluvieux est celui d'août avec une moyenne de $19 \pm 3 \mathrm{~mm}$. Le cumul pluviométrique relevé aux mois de juillet et d'août dans la station d'étude représente environ $100 \%$ du cumul annuel, soit $34 \pm 3,60 \mathrm{~mm}$. La composition floristique est composée d'espèces annuelles et pérennes Au total, 21 espèces végétales appartenant à 14 familles différentes ont été identifiées la vallée Tafidet, Les espèces pérennes (arbres et arbustes) représentent $47,82 \%$ et les espèces annuelles et bisannuelles représentent $52,18 \%$. Parmi les arbres, le genre Acacia est le plus dominant et représente $90 \%$.

Structure et composition zoologique de prétraitement : Des pièges en tube ont été utilisés, pour rendre compte de l'abondance relative des insectes marcheurs. La méthode de piégeage a consisté à placer 90 pièges en tubes sur trois transects parallèles, à raison de 30 pièges/transect. Les tubes en polypropylène (long $=11,5 \mathrm{~cm} ; \varnothing=4,5 \mathrm{~cm}$; volume $=175 \mathrm{ml}$ ) contenaient rapport aux risques d'exposition en conditions naturelles.

préalablement $10 \mathrm{ml}$ de formaline à $0,5 \%$ (liquide conservateur qui empêcherait le gonflement des invertébrés piégés), $10 \mathrm{ml}$ de liquide détergent (la mousse réduirait l'évaporation) et $90 \mathrm{ml}$ d'eau courante. Les transects sont distants de $20 \mathrm{~m}$ et les tubes sont distants entre eux de $5 \mathrm{~m}$. L'intervalle de suivi de trois jours a été utilisé ; l'expérimentation a duré 30 jours. Les insectes collectés ont été triés par groupe taxonomique pour des identifications au Centre Régional Agrhymet de Niamey et au British Muséum (Angleterre). Méthode d'évaluation de l'impact des pesticides sur les fourmis : L'évaluation de l'abondance relative des coléoptères a été effectuée avec des pièges en tube. L'utilisation des tubes ou pitfall est la méthode standard utilisée pour l'évaluation de l'abondance relative des arthropodes terrestres surtout les insectes marcheurs (Boer, 1977 ; Peveling et al., 1999; Wiktelius et al., 1999 ; Duncan et al., 2002). La méthode de piégeage a consisté à placer 90 pièges en tubes sur trois transects parallèles, à raison de 30 pièges/transect. Les tubes en polypropylène (long $=11,5 \mathrm{~cm} ; \varnothing=4,5 \mathrm{~cm}$; volume $=$ $175 \mathrm{ml}$ ) contenaient préalablement $10 \mathrm{ml}$ de formaline à $0,5 \%$ (liquide conservateur qui empêcherait le gonflement des invertébrés piégés), $10 \mathrm{ml}$ de liquide détergent (la mousse réduirait l'évaporation) et $90 \mathrm{ml}$ d'eau courante. Les transects sont distants de $20 \mathrm{~m}$ et les tubes sont distants entre eux de $5 \mathrm{~m}$. Les pièges ont été enterrés dans le sol en prenant soin d'ajuster leur bord supérieur au ras du sol. L'emplacement de chaque tube a été matérialisé par un piquet métallique. Dans chaque unité expérimentale, les pièges distants de $5 \mathrm{~m}$ sont disposés le long des diagonales ( 20 pièges/diagonale), en partant de $5 \mathrm{~m}$ du centre pour éviter l'effet du centre. Les cadences de suivi ont été de 4 jours correspondant à un optimum de collecte, vérifié par des essais préliminaires. L'ensemble de l'intervalle de suivi a été de 44 jours (4 jours avant les traitements et 40 jours après les traitements) soit -4 JAT (4 jours avant les traitements), 0 JAT (juste avant les traitements), 1 JAT (un jour après les traitements), 4 JAT, 8 JAT, 12 JAT, 16 JAT, 20 JAT, 24 JAT, 28 JAT, 32 JAT, 36 JAT et 40 JAT. L'intervalle de suivi post-traitement a été scindé en trois périodes pour rendre compte des effets des pesticides les effets des pesticides sur les fourmis à court terme (I), moyen terme (II) et long terme (III), comme suit : période I (1-12 jours) ; période II (16-24 jours) et période III (28-40 jours). A chaque intervalle de temps de suivi, le contenu du tube a 


\section{Abdou et Ahmed. J. Appl. Biosci. Évaluation des effets des pesticides utilisés en lutte chimique contre le Criquet pèlerin sur les fourmis au Niger.}

été vidé et remplacé. Les insectes ainsi collectés ont été triés par groupe taxonomique, comptés, et leurs échantillons conservés dans de l'éthanol à $70^{\circ}$ pour des identifications ultérieures au Centre Régional Agrhymet de Niamey (Niger) et au British Muséum (Angleterre).

Application des pesticides: Les applications des pesticides (en formulation ultra-bas volume) en couverture totale, ont été effectuées selon les recommandations techniques de la FAO (2001) avec un appareil à disque rotatif porté à la main du type MicroUlva ${ }^{\circledR}$. L'appareil est muni de cinq piles alcalines de 1,5 volts chacune, cette charge produirait un nombre de tours/minute de 6000 à 6400 selon les spécifications techniques du constructeur de l'appareil. Les doses de $225 \mathrm{~g} \mathrm{~m}$.a/ha de chlorpyriphos-éthyl et de $450 \mathrm{~g} \mathrm{~m}$.a/ha de fénitrothion ont été utilisées conformément aux recommandations de la FAO (1998) en lutte contre le Criquet pèlerin. Tous les traitements ont été effectués entre $6 \mathrm{~h}$ et $9 \mathrm{~h}$ du matin, lorsque la température était relativement basse. En effet, des courants d'air ascendants (courant de convection) peuvent entraîner la pulvérisation en dehors de la zone cible lorsque la température est supérieure à $35^{\circ} \mathrm{C}$. La hauteur d'émission a été de $1 \mathrm{~m}$, et la largeur de travail (andain) a été de $10 \mathrm{~m}$.

Dispositif expérimental: Le dispositif expérimental utilisé est le plan en bloc aléatoire complet (BAC). Trois blocs ont été disposés, distants entre eux de $1000 \mathrm{~m}$, et de $500 \mathrm{~m}$ entre les unités expérimentales qui sont des carrés de 16 hectares ( $400 \mathrm{~m} \times 400 \mathrm{~m}$ ). Trois traitements ont été constitués: les parcelles traitées au Chlorpyriphos-éthyl à la dose de $225 \mathrm{~g} \mathrm{~m}$.a/ha ; celles traitées au Fénitrothion à la dose de $450 \mathrm{~g} \mathrm{~m}$.a/ha et celles n'ayant pas reçu des pesticides ont constitué les témoins. Les blocs ont été disposés perpendiculairement au gradient d'hétérogénéité (végétation) qui diminue du nord au sud-est dans la vallée du Tafidet.

Analyse des données : L'analyse de la variance a été choisie pour effectuer une comparaison statistique basée sur le principe $\mathrm{BACl}$ (Avant-Après-Contrôle-Impact) (Stewart-Oaten et al., 1986 ; Underwood, 1991 ; Bence et al., 1996), suivi des tests de comparaisons multiples de Student-Newman-Keuls si l'hypothèse nulle $\mathrm{H}_{0}$ est rejetée au niveau $a=0,05$. Par souci de validité statistique, les observations avant et après les traitements doivent être effectuées dans chaque unité expérimentale.
Les valeurs exprimées en nombres absolus ont été transformées par la relation $y=\log (1+x)$ et celles exprimées en pourcentages ont subi une transformation de forme

$$
y=\arcsin \sqrt{x}
$$

en vue d'homogénéiser les variances et d'assurer une normalité aux distributions désaxées vers la droite (Sokal et Rholf, 1981, 1995). La valeur de $0 \%$ a été remplacée par $1 / 4 n$ et la valeur $100 \%$ par $100-1 / 4 n$, où $n$ représente le dénominateur utilisé pour calculer le pourcentage, c'est-à-dire, la taille de la population de référence.

Pour distinguer les effets des pesticides sur la faune noncible à court terme (I), moyen terme (II) et long terme (III), les périodes post-traitement ont été divisées en trois intervalles de temps.

Pour évaluer l'abondance relative d'une espèce donnée, pour chaque intervalle de temps, le nombre total des captures (TC) par espèce a été calculé. Par exemple, $\mathrm{TC}_{1}$ (capture totale au jour 1 ) $=30 ; \mathrm{TC}_{4}$ (capture totale au jour 4$)=17 ; \mathrm{TC}_{8}=9$ et $\mathrm{TC}_{12}=6$; donc pour la période $\mathrm{I}$ : $\mathrm{TC}_{\text {phase } 1}=(30+17+9+6) / 4=15,5$

Le paramètre d'effet $(C)$ utilisé (pour apprécier la fluctuation de la population) dans l'analyse statistique est la différence en abondance exprimée comme suit :

$$
C=\log \left(\mathrm{TC}_{\text {après }}+1\right)-\log \left(\mathrm{TC}_{\text {avant }}+1\right)
$$

Les valeurs négatives de $C$ indiquent une diminution de la population et les valeurs positives, une augmentation par rapport à la population initiale. Les données manquantes ont été corrigées en les multipliant par $40(40-\mathrm{m})$ où $\mathrm{m}$ représente le nombre de pièges manquants $\left(m_{\max }=4\right)$ (Peveling et al., 1999). L'effet des pesticides (E) pour chaque phase (I, II et III) et chaque espèce a été calculé (Henderson et Tilton, 1955 ; Peveling et al., 1999) :

$E(\%)=100\left[1-\left(\left(C_{b} \cdot T_{a}\right) /\left(C_{a} \cdot T_{b}\right)\right)\right]$

Où $\mathrm{C}_{b}\left(T_{b}\right)=$ la somme des captures dans toutes les unités expérimentales témoins (avant traitement) et $\mathrm{C}_{\mathrm{a}}\left(\mathrm{T}_{\mathrm{a}}\right)$, la somme des captures après les traitements dans les phases I, II et III.

Les risques écotoxicologiques ont été classés selon la FAO (1998), ils sont considérés hauts (> 75\%), moyens $(25-75 \%)$ et bas $(<25 \%)$. 


\section{Abdou et Ahmed. J. Appl. Biosci. Évaluation des effets des pesticides utilisés en lutte chimique contre le Criquet pèlerin sur les fourmis au Niger.}

\section{RÉSULTATS ET DISCUSSION}

En analysant les résultats par groupe taxonomique, un premier constant s'impose, la prépondérance des fourmis des genres Polyergus (38,38\%), Trachymyrmex (4,64\%) et Camponotus $(25,75 \%)$ Du point de vue quantitatif, les fourmis avec $68,77 \%$ des captures totales constituent le groupe zoologique le plus important (Figure 1). Ceci n'est pas surprenant puisque, leur présence dans la totalité des écosystèmes terrestres, et le fait qu'elles constituent

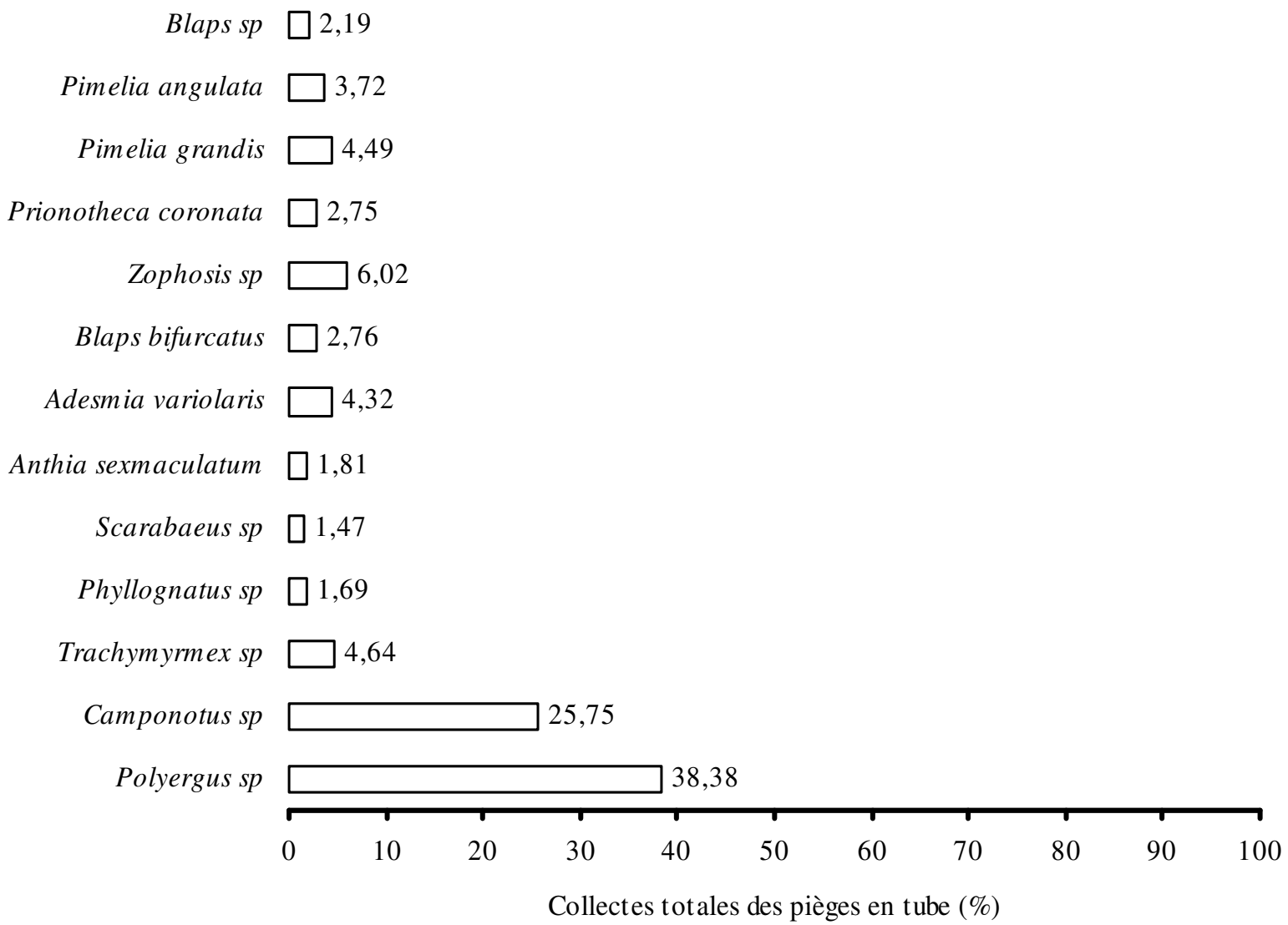

Figure 1: Composition et structure moyenne des insectes capturés avec les pièges en tube pendant la période d'observation dans la vallée du Tafidet (2004-06).

Impact des pesticides sur les fourmis (Hymenoptera : Formicidae) : Pendant la période de l'expérimentation qui s'est étalée de 2004 à 2006, $47315 \pm 1081$ individus, dont $56 \%$ constitués de Polyergus sp., $31 \%$ de Trachymyrmex sp. et $13 \%$ de Camponotus sp ont été capturés. L'abondance relative des fourmis a baissé brusquement un jour après les applications des pesticides (effet de choc), alors qu'elle a augmenté dans les parcelles témoins (Figures 2-4). Le chlorpyriphoséthyl et le fénitrothion ont un effet très nocif sur les une part importante de la biomasse animale, reflètent l'importance de leur succès écologique (Edward et Hölldobler, 2005). Les analyses statistiques réalisées ont montré qu'il n'y avait pas de différence significative entre les captures réalisées durant ces trois années $(p=$ $0,468)$. En revanche, la différence des captures interespèces était significative $F(13,26)=470 ; p=0,002$. 


\section{Abdou et Ahmed. J. Appl. Biosci. Évaluation des effets des pesticides utilisés en lutte chimique contre le Criquet pèlerin sur les fourmis au Niger.}

0,001 avec Polyergus sp., $F(2,6)=311,17 ; p=0,001$ avec Trachymyrmex sp. et $F(2,6)=345,33 ; p=0,001$ avec Camponotus sp. La réduction des populations des fourmis est très importante (> $98 \%)$. Dans la phase II (16-24 jours), le nombre de captures des différentes espèces a diminué (Figures 2-4) comparativement à la phase I. La plus importante réduction (> 75\%) est observée sur Camponotus sp. Par contre, avec Trachymyrmex sp. et Polyergus sp, cette réduction est considérée comme moyenne avec des taux compris entre $25 \%$ et $50 \%$. De manière générale, l'analyse statistique met en évidence une différence significative entre les traitements dans cette phase $\| ; F(2,6)=59,56 ; p=0,001$ avec Polyergus sp., $F(2,6)=241,83 ; p=0,001$ avec Camponotus sp. et $F(2,6)=512,64 ; p=0,001$ avec Trachymyrmex sp. De 28-40 jours après les traitements, le nombre de captures de différentes espèces de fourmis est faible (Figures 2-4) dans toutes les unités expérimentales y compris dans les parcelles témoins. La température habituellement basse observée à cette période, pourrait en être une des causes probables de cette réduction de captures enregistrée.

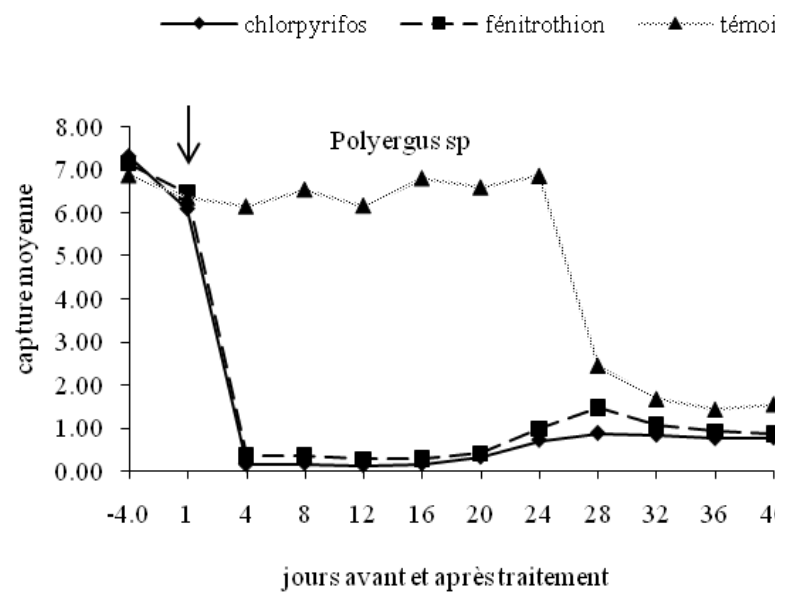

(a)

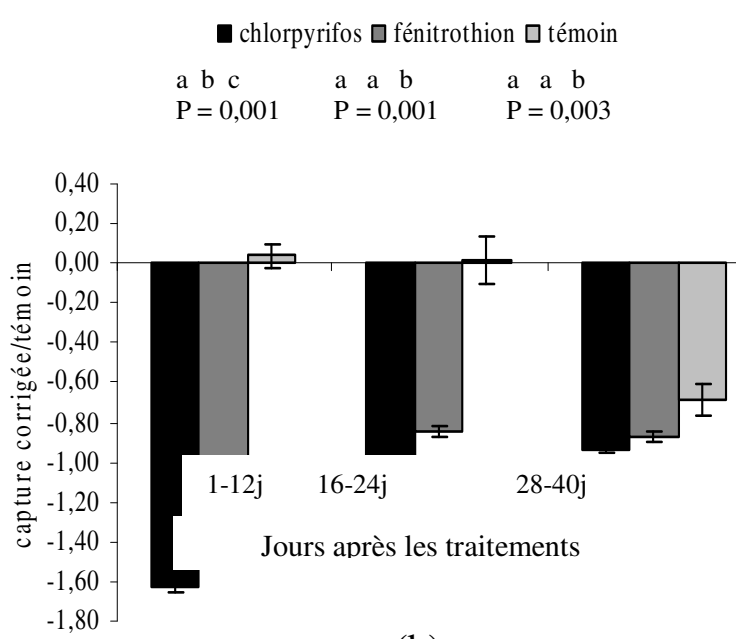

(b)

Figure 2: Capture moyenne de Polyergus $\mathrm{sp}$. avant et après traitement (a) et capture post-traitement corrigée ( $\pm \mathrm{SE})$ par rapport au témoin (b). Les valeurs avec la même lettre ne sont pas significativement différentes au niveau $\alpha=0,05$. La flèche indique le jour des traitements.

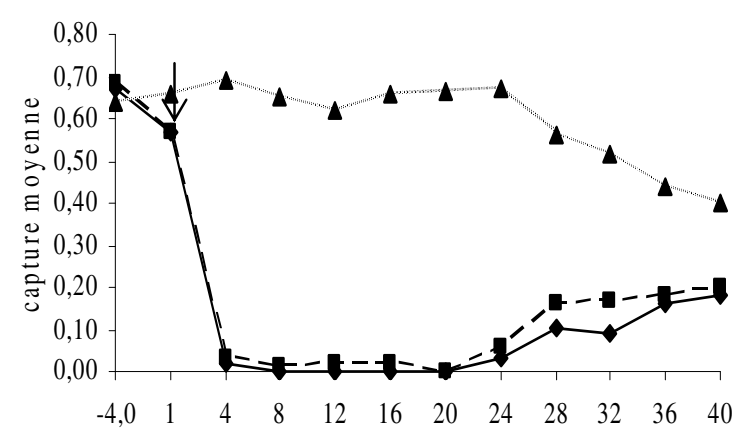

(a)

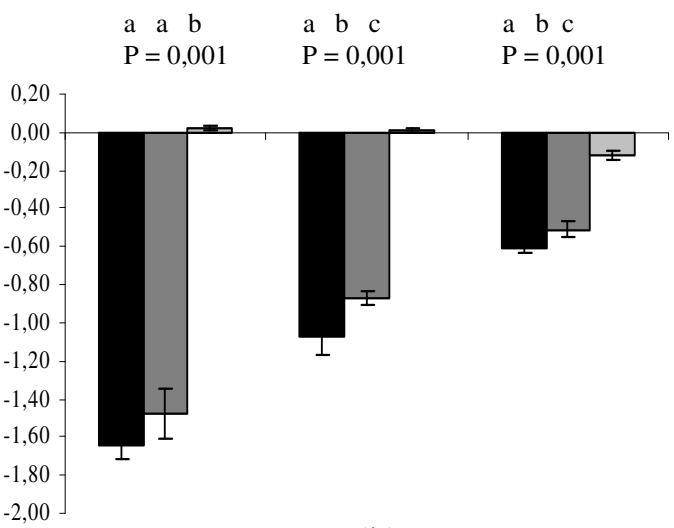

(b) 


\section{Abdou et Ahmed. J. Appl. Biosci. Évaluation des effets des pesticides utilisés en lutte chimique contre le Criquet pèlerin sur les fourmis au Niger.}

Figure 3 : Capture moyenne de Camponotus $\mathrm{sp}$. avant et après traitement (a) et capture post-traitement corrigée ( $\pm \mathrm{SE})$ par rapport au témoin (b). Les valeurs avec la même lettre ne sont pas significativement différentes au niveau $a=0,05$. La flèche indique le jour des traitements.

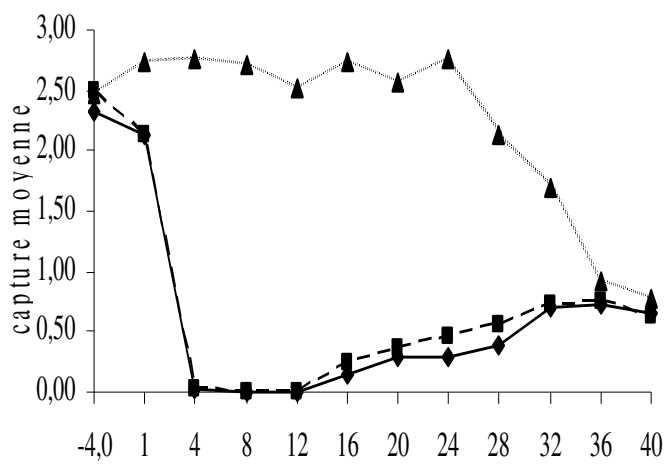

(a)

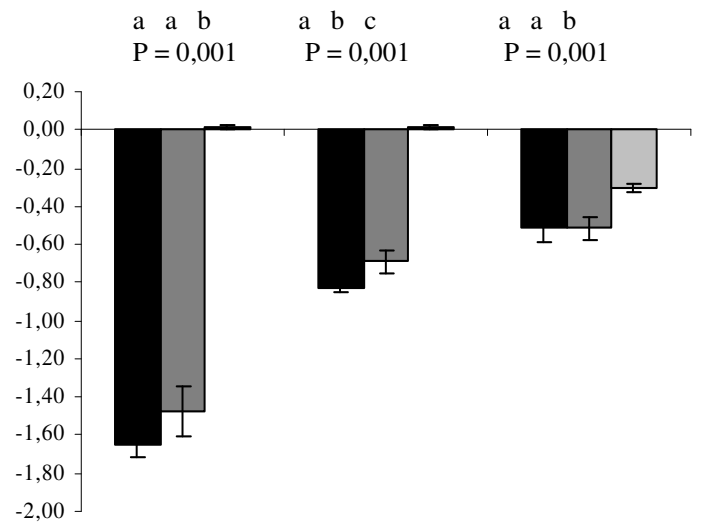

(b)

Figure 4: Capture moyenne de Trachymyrrmex sp. avant et après traitement (a) et capture post-traitement corrigée $( \pm S E)$ par rapport au témoin $(b)$. Les valeurs avec la même lettre ne sont pas significativement différentes au niveau $a=$ 0,05 . La flèche indique le jour des traitements.

\section{CONCLUSION}

De manière générale, les pesticides de synthèse utilisés en lutte antiacridienne ne sont pas spécifiques aux seuls acridiens. Ils ont souvent un effet réducteur sur les ennemis naturels des acridiens, sur des oiseaux et sur des insectes d'utilités agronomiques (Peveling et al., 2003, 1999; Mullié et Keith, 1993; Balança et de Visscher, 1997 ; Mamadou et al., 2005). En effet, le chlorpyriphos-éthyl et le fénitrothion utilisés respectivement aux doses de $225 \mathrm{~g} \mathrm{~m}$.a/ha et de $450 \mathrm{~g}$ $\mathrm{m}$.a/ha, ont un effet négatif sur l'abondance relative des fourmis. Des réductions importantes de populations des

\section{REMERCIEMENTS}

Les auteurs remercient le Comite FAO de lutte contre le Criquet pèlerin (DLCC) qui a entièrement financé cette étude dans le cadre d'une thèse de doctorat. Nous tenons à exprimer toute notre gratitude à la Commission de lutte contre le criquet pèlerin dans la région occidentale (CLCPRO) qui a attribué cette bourse au

\section{RÉFÉRENCES BIBLIOGRAPHIQUES}

Anthelme F, Waziri-Mato M, de Boissieu D., Giazzi F., 2006. Dégradation des ressources végétales au contact des activités humaines et perspectives de conservation dans le massif de l'Aïr (Sahara, Niger). VertigO 7 (2): 1-12. fourmis de plus de $98 \%$ en présence de deux insecticides ont été notées à 12 jours après les traitements. Dans l'intervalle de temps 16-24 jours, le nombre de capture de ces trois espèces a diminué par rapport à l'intervalle 1-12 jour après les traitements, un taux de réduction de $75 \%$ avec le genre Camponotus sp et un taux de réduction compris entre $25-50 \%$ ont été notés respectivement avec Trachymyrmex sp et Polyergus sp. De $28-40$ jours après les traitements, le nombre de captures de différentes espèces de fourmis est faible dans toutes les unités expérimentales y compris dans les parcelles témoins.

Niger. Nous remercions la Direction de la Protection des Végétaux pour son aide dans la logistique qui nous a permis de mener à bien ces travaux. Enfin, nous remercions tous ceux qui ont contribué à la réalisation de cette étude.

Anthelme F, Michalet R, Saadou M, 2007. Positive associations involving the tussock grass Panicum turgidum Forssk. in the Aïr Ténéré Réserve (Sahara, Niger). Journal of Arid Environment 68: 348-362. 


\section{Abdou et Ahmed. J. Appl. Biosci. Évaluation des effets des pesticides utilisés en lutte chimique contre le Criquet pèlerin sur les fourmis au Niger.}

Arbuckle T.A., Lin Z., Mery S.L., 2001. An exploratory Analysis of the effect of Pesticide Exposure on the Risk of Spontaneous Abortion in an Ontario Farm Population," Environmental Health Perspective, 109 (8): 801-807.

Balança G, de Visscher MN, 1997. Impacts on Nontarget Insects of a New Insecticide Compound used Against Desert Locust (Schistocerca gregaria Forskal, 1775). Archives of Environmental Contamination and Toxicology 32: 58-62.

Bashir MO, El Rahim Sorkati IA, Hassanali A, 1998. The effect of desert locust, Schistocerca gregaria (Forskal), on the productivity of rangeland in Red Sea coast of The Sudan and its population management through environment-friendly control tactics. In: Squire, V.R. \& Sidahmed, A.E. (eds.), Drylands: Sustainable use of rangeland into the twenty-first century. IFAD SERIES. Technical Reports, pp. 321-328.

Bence JR, Stewart-Oaten A, Schroeter SC, 1996. Estimating the size of an effect from a beforafter-control-impact paired series design. In: Schmitt RJ and Osenberg CW (eds) Detecting ecological impacts: concepts and application in coastalhabitats. Academic Press, USA.

Boer PJ, 1977. Dispersal power and survival. Carabids in cultivated countryside. Miscellaneous Paper Landbouwhogeschool Wageningen 14: 1-190.

Clavel J., 1996. "Farming, pesticide use and hairy-cell leukemia", Scandinavian Journal of Work, Environmental and Health, 22: 285-293.

David G., 2001. Effets sublétaux de l'imidaclopride sur le comportement de l'abeille domestique (Apis mellifera). Thèse. Université Pierre et Marie Curie, France, $124 \mathrm{p}$.

Dinkins R.L., Brazzel J.R., Wilson C.A., 1971. Effects of early season insecticide applications on major predaceous arthropods in cotton fields under an integrated control program. J. Econom. Entomol., 64 (2): 480-484.

DMN, 2005. Climatologie du Niger. [en ligne].

http://www.meteo-niger.net/html/climatccl2.htm (Page consultée le 17 décembre 2006).

Duncan D.F., Krasnov B., McMaster M., 2002. Novel case of tenebrionid beetle using discountinuous gas exchange cycle when dehydrated. Physiological entomology, 27: 79-83.

Edward OW, Hölldobler B, 2005. The rise of ants: A phylogenetic and ecological explanation. Ecology Evolution Phylogeny and Sociology 102 (21): 7411-7414.
FAO, 1997. Évaluation des essais de terrain relatif à l'efficacité des insecticides sur les criquets et les sautériaux. Rapport de la FAO du Groupe Consultatif sur les Pesticides. Sixième réunion, Rome du 10 au 12 décembre 1996, 21 p.

FAO, 1998. Évaluation of field trial data on the efficacy and selectivity of insecticides on locust and grasshoppers. Report to FAO by the Pesticide Referee Group. $7^{\text {th }}$ meeting, Rome, 2-6 March 1998, $24 \mathrm{p}$.

FAO, 2001. Directives sur le Criquet pèlerin. Lutte antiacridienne. 2è Edition, $41 \mathrm{p}$.

FAO/PAM, 2004. Mission FAO/PAM d'évaluation des récoltes et des disponibilités alimentaires au Niger. Rapport Spécial, 21 décembre 2004.

Giazzi F, 1993. Ressources Naturelles et aménagement du milieu dans le massif de l'Air (Niger). Actes du Gème colloque Franco-japonais de géographie : « environnementetaménagements montagnards », Grenoble 16-20 septembre 1991, IGA. DRGA n¹3, pp. 171-176.

Giazzi F, 1996. Étude initiale. La Réserve Naturelle Nationale de l'Aïr et du Ténéré (Niger). La connaissance des éléments du milieu naturel et humain dans le cadre d'orientations pour un aménagement et une conservation durables. Analyse Descriptive. MH/E, WWF, UICN, 712p.

Henderson CF, Tilton EW, 1955. Test with acaricides against the brown wheat mite. Journal of Economic Entomology 48: 143-157.

Karfu KJ, 1998Effects of ant exclusion during outbreaks of a defoliator and a sap-sucker on birch. Ecology and Entomology 23: 185-194.

Kogo SA, Krall S, 1997. Yield losses on pearl millet panicles due to grasshoppers: a new assessment method. In: Krall S., Peveling R., Ba Diallo D. (Eds), New Strategies in Locust Control. Birkhäuser, Basle, Switzerland, pp. 415423.

Krall S, 1994. Importance of locusts and grasshoppers for African agriculture and methods for determining crop losses. In Krall, S. Wilps, H. (Eds.), New Trends in Locust Control. Schriftenreihe der GTZ 245, TZVerlagsgeselschaft, Germany: Rossodorf, $72 \mathrm{p}$.

Lecoq M., 2001. Recent progress in Desert and Migratory Locust management in Africa. Are preventative actions possible ? Journal of Orthopteran Research, 10 (2): 277-291.

Liu F., Chen J., Chai J., Zhang X., Bai D., Roubik D.W., 2007. Adaptative functions of defensive plant 


\section{Abdou et Ahmed. J. Appl. Biosci. Évaluation des effets des pesticides utilisés en lutte chimique contre le Criquet pèlerin sur les fourmis au Niger.}

phenolics and non-linear bee response to nectar components. Functional Ecology, 21: 96-100.

Levine R., 1991. Recognized and possible effects of pesticides in humans. In W.J. Hayes \& E.R. Laws : Handbook of Pesticide Toxicology. Academic Press, San Diego, CA, USA, 275-360.

Mamadou A, Mazih A, Ghaout S, Hormatallah A, 2005. Étude de l'impact des pesticides utilisés en lutte contre le Criquet pèlerin (Schistocerca gregaria Forskål, 1775) (Acrididae : Orthoptera) sur deux espèces de Prionyx (Hymenoptera, Sphecidae) dans l'Aïr (Niger). Actes Institut Agronomique et Vétérinaire 25 (1-2) : 59-65.

Mullie WC, Keith J., 1993. The effects of aerially applied fenitrothion and chlorpyriphos on birds in the savannah of northern Senegal. Journal of Applied Ecology 30: 536-550.

Niemela P, Haila H, Halme E, Pajunen T, Punttila P, 1992. Small scale heterogeneity in the spatial distributions of carabid beetles in the Southern Finnish taiga. Journal of Biogeography 19: 173181.

OMS/PNUE, 1989. Public health impact of pesticides used in agriculture. Report of WHO/UNEP Working Group, Genève, $140 \mathrm{p}$.

Peveling R, Attignon S, Langewald J, Ouambama Z, 1999. An assessment of the impact of biological and chemical grasshopper control agents on ground-dwelling arthropods in Niger based on presence/absence sampling. Crop Protection 18: 323-339.

Peveling R, McWilliam AN, Nagel P, Rasolomanana H., Raholijoana, Rakotomianina L., Ravoninjatovo A., Dewhurst C.F., Gibson G., Rafanomezana S., Tingle C.C.D., 2003. Impact of locust control on harvester termites and endemic vertebrate predators in Madagascar. Journal of Applied Ecology 40: 729-741.

Pimentel D., McLaughlin, Zepp A., Lakitan B., Kleinman P., Vancini F., Roach W.J., Graap E., Keeton W.S., Seig G., 1991. Environmental and economic effects of reducing pesticide use. Bioscience, 41: 402-409.

Predtetchensky SA, 1935a. Données sur l'étude du Criquet pèlerin (Schistocerca gregaria Forsk.) en Asie Centrale et en Transcaucasie (invasions de 1929 et 1930). Trudy po Zastchite Rasteniï. Série I : Entomologie 11: 1-92.

Punttila P, Niemela P, Karfu KJ, 2004. The impact of wood ants (Hymenoptera: Formicidae) on the structure of invertebrate community on mountain birch (Betula pubescens ssp). Annales Zoologici Fennici 41: 429-446

Roger C., Coderre D., Vincent C., 1991. Apparent mortality of Coleomegilla maculata (Coccinellidae) following pesticide treatment: possibility of overlooking predators. In: Polgar L., Chambers R.J., Dixon A.F.G., Hodek I. (Eds.) Behaviour and Impact of Aphidophaga, pp. 329336.

Römbke J, Moltmann JF, 1996. Applied Ecotoxicology. Boca Raton : CRS Press, $282 \mathrm{p}$.

Sokal RR., Rholf FJ, 1981. Biometry. The Principe and practice of statistics in biological research. $2^{\text {nd }}$ edition. Freeman and Co. New York, $859 \mathrm{p}$.

Roubik D.W., 2002. Feral African bees augment neotropical coffee yield. IN: Kevan P \& Imperatriz Fonseca VL (eds) - Pollinating Bees The Conservation Link Between Agriculture and Nature - Ministry of Environment (Brasilia). p. $255-266$

Sokal RR, Rohlf FJ, 1995. Biometry. $3^{\text {rd }}$ edition. W.H. Freeman, San Francisco.

Savolainen R, Vepsalainen K, Wuorenrinne H, 1989. Ant assemblages in the taiga biome: testing the role of territorial wood ants. Oecologia 81: 481-486.

Stewart-Oaten A, Murdoch WW, Parker KR, 1986. Environmental impact assessment: "pseudoreplication" in time? Ecology 67 (4): 929940.

Thiollet-Scholtus M, 2004. Contribution d'un indicateur de qualité des eaux de surface vis-à-vis des produits phytosanitaire à l'échelle $d u$ bassin versant viticole. Thèse de doctorat. Institut National Polytechnique de Lorraine (France), $206 \mathrm{p}$.

Underwood AJ, 1991. Beyond BACl: experimental designs for testing human environmental impacts on temporal variations in natural populations. Australian Journal of Marine and Freshwater Research 42: 569-587.

UNESCO, 1996. Convention concernant la protection du Patrimoine Mondial Culturel et Naturel. Comité de Patrimoine Mondial. Dix-neuvième session, Berlin, Allemagne, 4-9 décembre 1995, $124 \mathrm{p}$.

Whalon M.E., Elsmer E.A., 1982. Impact of insecticides in Illioia pepperi and its predators. J. Econom. Entomol., 75: 356-358.

Zimmermann Y., Roubik D.W., Eltz T., 2006. Speciesspecific attraction to pheromonal analogues in 
Abdou et Ahmed. J. Appl. Biosci. Évaluation des effets des pesticides utilisés en lutte chimique contre le Criquet pèlerin sur les fourmis au Niger.

orchid bees. Behav. Ecol. Sociobiol., 60: 833-

843. 